\title{
A Comparison of Selected Algorithms of Form Deviation Calculation
}

\author{
Marek MAGDZIAK
}

\begin{abstract}
The article concerns the verification of accuracy of selected algorithms of form deviation evaluation. The paper presents the results of experimental investigations concerning coordinate measurements of products composed of free-form surfaces, which are characterized by various degrees of geometric complexity. The experimental investigations were conducted with the use of the method of form deviation estimation which is based on the Lagrange interpolation of measured points and two additional algorithms available in the Calypso software, which cooperates with a coordinate measuring machine. Moreover, the experimental research was performed by using of the coordinate measuring machine ACCURA II equipped with the scanning probe VAST XT. Based on the analysis of gained results of coordinate measurements the method of form deviation calculation which uses the Lagrange interpolation of measured points in order to calculate an actual curve achieved better results than one of the analysed algorithms of the Calypso inspection software.
\end{abstract}

Keywords: coordinate measuring technique; form deviation; free-form surface; interpolation

\section{INTRODUCTION}

There can be distinguished a number of factors which influence the accuracy of measurements conducted with the use of various coordinate measuring systems. The accuracy of coordinate measurements of products characterized by regular geometric shapes or composed of curvilinear surfaces depends on e.g. an applied measurement strategy. A measurement strategy in the coordinate measuring technique may include, for example, specific methods of a probe radius correction process [1] and localization of measured points $[2,3]$. The distribution of measured points is one of the fundamental steps which should be taken into account at the beginning of coordinate measurements.

There are a lot of methods of a distribution of measured points or scanning lines along which measurement processes of free-form surfaces of measured objects are conducted. The localization of measured points should take into account the parts of a measured product which are characterized by the lowest quality of manufacturing. The wrong methods of arrangement of measured points may lead to:

- long-time of realization of a given measurement task;

- bypassing parts of a surface which are characterized by the biggest errors of manufacturing;

- improper computation of an associated feature.

Examples of two strategies of measurements concerning free-form surfaces are shown in the paper by ElKott and Veldhuis [4] as well as in the work by ElKott [5]. Both publications concern the selection of locations of scanning lines on surfaces of measured objects to be applied when a CMM with a measuring probe working in the scanning mode is planned to be used. Two methods for determining the arrangement of target scanning lines are proposed:

- the method named automatic sampling, considering maximum differences between a substitute model and a CAD model;

- the method making use of information about the change of average curvature of a measured free-form surface.
Both methods are limited by a maximum number of scanning lines and a tolerance concerning the accuracy of computing substitute geometry. Those limitations are defined by a user at the beginning of each algorithm. The substitute model is generated based on distributed scanning lines.

In the works by ElKott et al. [6] and ElKott [7] four algorithms for sampling free-form surfaces are presented. The first one consists in the uniform distribution of measured points in individual segments along accepted surface directions of a measured object and can be used in the case of measurements of curvilinear surfaces which are characterized by a small change of curvature. The second algorithm is based on the division of a given surface on surface patches. Information concerning the division of a given surface into surface patches is taken from the knot vector. Next, there are calculated the areas of each surface patch. The patches are sorted with consideration to their area. The patch with the biggest area becomes in the second algorithm the one with the biggest number of measured points. There exists, however, some risk that small surface patches with significant changes of shape may be omitted. The next algorithm - the third one - for computing a distribution of measured points creates a list of surface patches sorted with consideration to the calculated average curvature of a patch. Analogously to the second algorithm the patch with the biggest curvature becomes in the third algorithm the one with the biggest number of measured points. The last algorithm presented by ElKott et al. [6] and ElKott [7] - the fourth one - links two previous algorithms which are based on the area of a surface patch and its average curvature. That algorithm enables defining socalled weight coefficients for each criterion (patch area, average curvature) in order to enlarge the role of one approach in relation to the other, depending on the character of observed changes of a shape of a measured free-form surface of a given object.

Mingrang Y. et al. [8] presented the method of a distribution of measured points on free-form surfaces which takes into account the quality of manufactured products. The authors pointed out that the disadvantage of most available algorithms of localization of measured points is that they are based on nominal points. Therefore, the algorithm of localization of measured points uses the 
form error model which takes into account deviations associated with the inaccuracy of a manufacturing process of a product. In order to construct the form error model the maximum deviation equal to $0.1 \mathrm{~mm}$ was used. The form error model was constructed by adding form deviations to a nominal model of a workpiece. The added deviations were proportional to the curvatures of an analysed surface. The proposed method was compared to the uniform distribution of measured points and the algorithm based on the curvature of a measured surface. On the basis of the results of experimental investigations the presented method achieved better results than the other investigated methods. The better results were gained in the case of the time of measurements and the number of measured points. The presented algorithm of a distribution of measured points works in the iterative way and includes the following stages:

- the selection of some measured points distributed on a form error model near its edges;

- the construction of a substitute model and the comparison of a constructed model to a form error model;

- more measured points are added to the parts of measured surfaces which are characterized by the biggest form deviations.

In the paper by Barari [9] there was presented the algorithm of localization of measured points which takes into account the information about the manufacturing process of a product. The information is gained from the results of simulations conducted by using of computer aided manufacturing software. The proposed algorithm of a distribution of measured points includes the following stages:

- the simulation of a machining process of a product;

- the measurement of a machined product by using of a coordinate measuring machine;

- the best-fit of a machined surface of a product to measured points with the use of the method of least squares;

- the distribution of measured points taking into account the results of fitting.

After distributing measured points coordinate measurements of products can be conducted and form deviations of manufactured workpieces can be calculated. Therefore, the algorithms of a distribution of measured points and form deviation calculation do not need to be considered independently of each other. The methods of form deviation evaluation may supplement the existing algorithms of localization of points and consequently make the selection of the appropriate measurement strategy in the coordinate measuring technique much easier. The paper concerns the last stage of coordinate measurements which is connected with assessing the quality of a product.

The paper presents the results of experimental investigations regarding coordinate measurements of products. The main objective of the research was to determine the influence of an applied method of form deviation evaluation on results of real coordinate measurements of curvilinear surfaces of products. The research was performed with the application of different measurement strategies. The need of conducting detailed real investigations arose from the results of preliminary investigations which were presented by Magdziak [10]. The preliminary research concerns coordinate measurements of only one free-form surface of a measured object. Therefore the results had to be verified in the case of additional surfaces differing in their shape. Moreover, the paper includes the simple algorithm of improving the accuracy of a selected method of form deviation calculation which is available in commercial measurement software.

In the next part of the paper the analysed algorithms of form deviation calculation are presented. Moreover, the article presents the investigated curvilinear surfaces of measured objects, the applied measuring system and the results of experimental investigations. The measured surfaces are characterized by a various degree of geometric complexity. The results of experimental research were gained by using of three different algorithms of form deviation calculation and different measurement parameters. The research was conducted with the use of the algorithm of form deviation calculation which is based on the Lagrange interpolation of measured points and two additional algorithms available in selected commercial software of a coordinate measuring machine. The last sections of the paper concern the method of improving the accuracy of one of considered algorithms of form deviation evaluation and conclusions regarding the results of conducted research.

\section{ANALYSED ALGORITHMS OF FORM DEVIATION CALCULATION}

Two considered methods of form deviation evaluation are available in the Calypso inspection software of the Carl Zeiss company. Those methods can be selected from the 2D curve and 3D curve measurement elements of the mentioned metrological software. In the case of the first commercial method local deviations are measured at each nominal point in the direction of a normal vector up to the intersection with an actual curve, which represents corrected measured points (the method denoted as $\mathrm{N}$ in the next parts of the article). The disadvantage of this method is the possibility of calculation of wrong local deviations based on parts of measured curves resulting from an extrapolation process. The extrapolation of a measured curve may be conducted due to the missing group of measured points. The insufficient number of measurement data may result from filtering some points after starting the scanning process of a measured workpiece.

In the second commercial algorithm a local deviation is measured as the distance between the point located on an actual curve and a nominal curve. Therefore, this method enables calculation of form deviations based on measured points (the method denoted as R) [11]. On the basis of the results of numerical investigations, which were presented by Magdziak [10], the method $\mathrm{R}$ can be treated as the reference one. It means that the method $\mathrm{R}$ is the most accurate. The disadvantage of the considered inspection software is that the user has no possibility to easily implement other methods of form deviation evaluation, besides the default available.

The third analysed algorithm of form deviation calculation (the method denoted as L) is not available in 
the mentioned software. That method divides corrected measured points into groups composed of five points which are interpolated using the Lagrange and the Chebyshev interpolation methods. Therefore, there are constructed the groups of polynomial curves of a fourth degree, which represent the real shape of a measured workpiece. In the next stage, after calculating interpolation curves, the deviations between nominal points and intersection points are determined. The intersection points are created by using of interpolation curves and lines which are perpendicular to the measured profile of a workpiece at its nominal points [10].

In the paper the results of investigations were analysed using only the Lagrange interpolation method. The choice of the method of interpolation was made based on the results of statistical analysis [10], which did not reveal significant differences between two selected interpolation methods in the case of the considered measurement task. When using the Lagrange interpolation method and five interpolated corrected measured points with coordinates $\left(x_{i}, y_{i}\right)$ the interpolation function $L(x)$ is written with Eqs. 1 and 2 [10].

$$
\begin{aligned}
& L(x)=y_{0} L_{0}(x)+y_{1} L_{1}(x)+y_{2} L_{2}(x)+y_{3} L_{3}(x)+y_{4} L_{4}(x) \\
& \left\{\begin{array}{l}
L_{0}(x)=\frac{\left(x-x_{1}\right)\left(x-x_{2}\right)\left(x-x_{3}\right)\left(x-x_{4}\right)}{\left(x_{0}-x_{1}\right)\left(x_{0}-x_{2}\right)\left(x_{0}-x_{3}\right)\left(x_{0}-x_{4}\right)} \\
L_{1}(x)=\frac{\left(x-x_{0}\right)\left(x-x_{2}\right)\left(x-x_{3}\right)\left(x-x_{4}\right)}{\left(x_{1}-x_{0}\right)\left(x_{1}-x_{2}\right)\left(x_{1}-x_{3}\right)\left(x_{1}-x_{4}\right)} \\
L_{2}(x)=\frac{\left(x-x_{0}\right)\left(x-x_{1}\right)\left(x-x_{3}\right)\left(x-x_{4}\right)}{\left(x_{2}-x_{0}\right)\left(x_{2}-x_{1}\right)\left(x_{2}-x_{3}\right)\left(x_{2}-x_{4}\right)} \\
L_{3}(x)=\frac{\left(x-x_{0}\right)\left(x-x_{1}\right)\left(x-x_{2}\right)\left(x-x_{4}\right)}{\left(x_{3}-x_{0}\right)\left(x_{3}-x_{1}\right)\left(x_{3}-x_{2}\right)\left(x_{3}-x_{4}\right)} \\
L_{4}(x)=\frac{\left(x-x_{0}\right)\left(x-x_{1}\right)\left(x-x_{2}\right)\left(x-x_{3}\right)}{\left(x_{4}-x_{0}\right)\left(x_{4}-x_{1}\right)\left(x_{4}-x_{2}\right)\left(x_{4}-x_{3}\right)}
\end{array}\right.
\end{aligned}
$$

The advantage of the proposed method of form deviation calculation is the possibility of its implementation in most inspection software cooperating with a coordinate measuring machine. The method can be implemented in the Calypso software with the use of the parameter coded measurements (PCM) module. That module has the possibility of using external applications, which can take into account the proposed method [10]. In order to calculate interpolation curves, necessary for calculating form deviations, nominal points, measured points and nominal vectors can be exported from the 2D curve measurement element of the considered software. Such an element enables the coordinate measurements of products composed of free-form surfaces.

\section{MEASURED WORKPIECES}

The experimental investigations concern the coordinate measurements of products composed of freeform surfaces. For each considered object four free-form curves were extracted (Fig. 1). The measured workpieces had free-form surfaces with varying curvature. The measurements were conducted along extracted profiles (denoted as $\mathrm{c} 1-\mathrm{c} 4$ ). The measured products were made of an aluminium alloy and machined by using of the $\mathrm{CNC}$ machine tool DMU 100 monoBLOCK. The nominal models of workpieces were prepared using the CATIA V5 computer aided design software.

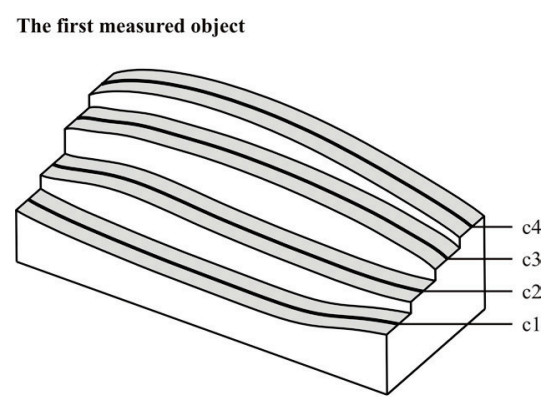

The second measured object

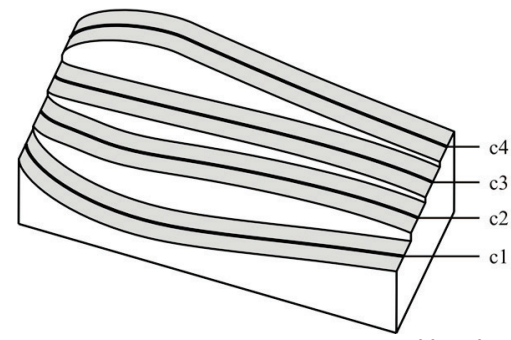

Figure 1 The measured products composed of free-form surfaces

\section{APPLIED COORDINATE MEASURING SYSTEM}

The coordinate measuring machine ACCURA II equipped with the measuring probe VAST XT and the Calypso inspection software was applied during coordinate measurements. The measuring system is characterized by the following accuracy parameters:

- $\quad E_{L, M P E}=(1,6+L / 333) \mu \mathrm{m}$

- $\quad P_{F T U, M P E}=1,7 \mu \mathrm{m}$

- $\quad M P E_{T i j}=2,5 \mu \mathrm{m}$

- $\quad M P T_{i i j}=50,0 \mathrm{~s}$

where: $E_{L, M P E}$ - a maximum permissible error of a length measurement; $L$ - a measured length, mm; $P_{F T U, M P E}-$ maximum permissible single-stylus form error; $M P E_{T i j}-\mathrm{a}$ maximum permissible scanning error; $M P T_{i i j}$ - maximum permissible scanning test duration.

Moreover, the acceleration of the applied CMM equal to $2 \mathrm{~m} / \mathrm{s}^{2}$ was used when analysing the results of real coordinate measurements. The results of measurements depend, among others, on the number and the distribution of measured points. Those components of a measurement strategy can be modified by declaring the following settings of the Calypso measurement software:

- start and end nominal points which have to be established when performing coordinate measurements in the scanning mode with the use of scanning probes. The change of the mentioned points is realized at the stage of defining a measurement strategy;

- the time of masking of measured points after starting a scanning process or before its end (Fig. 2);

- the number of points which are masked after starting a scanning process or before ending a measurement process (Fig. 2). 


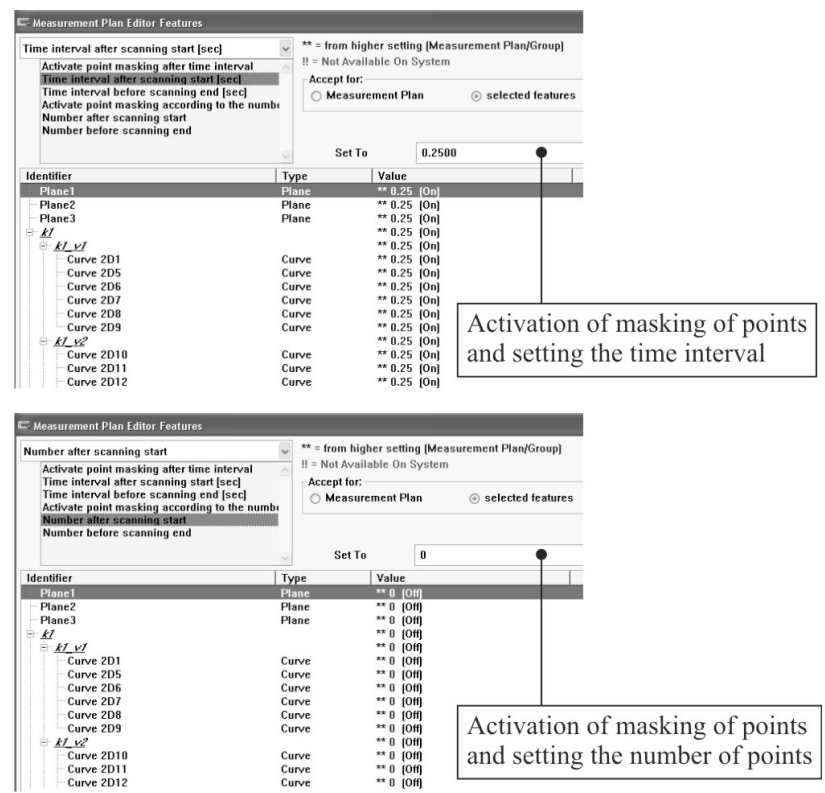

Figure 2 Activating the settings in the field of masking of measured points

The considered settings may cause the change of the shape of a measured profile which represents a measured free-form surface. The measured profile may differ from the real shape of a measured surface. In some cases the difference may be very significant. The wrong measured profile may result from the extrapolation process of a measured curve because of the insufficient number of measured points and it may increase the inaccuracy of coordinate measurements. In order to reduce such inconvenience some nominal points at which local deviations are wrongly calculated should be identified and deleted. The analysed inspection software does not detect automatically nominal points at which local deviations should not be evaluated. Wrongly calculated results can be removed only based on the analysis of outliers. Therefore, the considered metrological software should be supplemented with an external application which may help to detect the considered points in the automatic mode.

\section{RESULTS OF MEASUREMENTS}

The coordinate measurements were conducted by using of 2D curve measurement element. The measurements were performed for the selected curves located in the middle of the free-form surfaces of each measured object. The uniform distribution of nominal and measured points along measured profiles of analysed products was used. The measurements were performed with the use of different scanning velocities (denoted as $v_{s}$ ) and distances between measured points (denoted as $d$ ). The following measurement parameters were used during measurements:

- $\quad v_{s}=(10,20,30) \mathrm{mm} / \mathrm{s}$;

- $d=(0,5 ; 1,0 ; 1,5 ; 2,0 ; 2,5 ; 3,0) \mathrm{mm}$.

The applied scanning parameters, including a scanning speed and the number of measured points, are higher than those recommended by the producer of the used coordinate measuring system [12]. The selection of the parameters of a measurement strategy should be based most of all on the expected uncertainty of a coordinate measurement and the required time of measurements.

The radius of a stylus tip was equal to $1,5 \mathrm{~mm}$. Moreover, the coordinate measurements were conducted by using of the algorithms of form deviation calculation based on nominal points $(\mathrm{N})$, measured points $(\mathrm{R})$ and the Lagrange interpolation method (L). The main aim of applying different measurement parameters was to obtain various groups of measured points. The gained sets of points were approximated by actual curves which represent the real shape of measured workpieces. The results of performed experimental research are presented in Fig. 3. Based on the obtained form deviations the ranges of results were calculated.

On the basis of the achieved results there was confirmed significant impact of the applied measurement strategy, including a scanning speed and a distance between points, on the values of a form deviation calculated by using of the method $\mathrm{N}$. The maximum range of form deviations, gained using the method $\mathrm{N}$, was equal to $0,883 \mathrm{~mm}$. The applied measurement parameters do not substantially affect the results of measurements obtained using the method $\mathrm{L}$ based on the Lagrange interpolation algorithm. When using the method $\mathrm{L}$ the biggest range of results was achieved in the case of the second surface of the second workpiece. Moreover, the results gained using the method L, in most cases, are similar to those obtained with the use of the method $\mathrm{R}$ of deviation calculation.

\section{MODIFICATION OF A SET OF NOMINAL POINTS}

There was developed the method of increasing the accuracy of the method $\mathrm{N}$ of form deviation calculation. The developed algorithm is based on the modification of a set of nominal points at which local deviations are calculated. A set of nominal points should be modified because of the wrong shape of an actual curve which represents a measured object. The wrong shape may result from e.g. the applied time of masking of measured points.

Fig. 4 presents the measured curves obtained using the following parameters of measurements: $v_{s}=15 \mathrm{~mm} / \mathrm{s}, d=$ $0,5 \mathrm{~mm}$. The beginnings of the measured curves do not correspond to the real shapes of measured workpieces and they depend on the applied time of masking of measured points after starting a scanning process. In order to reduce such effect pre-travel distance may be applied when planning a scanning process. However, in some cases, taking into account e.g. the shape of a measured workpiece, it is not possible.

The proposed algorithm enables to specify local deviations which are wrongly calculated and should not be taken into account during the analysis of results of coordinate measurements. Consequently, those deviations should be removed from the set of measured local deviations in order to increase the accuracy of coordinate measurements of free-form surfaces of products. The developed algorithm takes into account such parameters of measurements as the acceleration of a probe, the scanning velocity, the distance between measured points and the time of masking of measured points after starting a scanning process. 

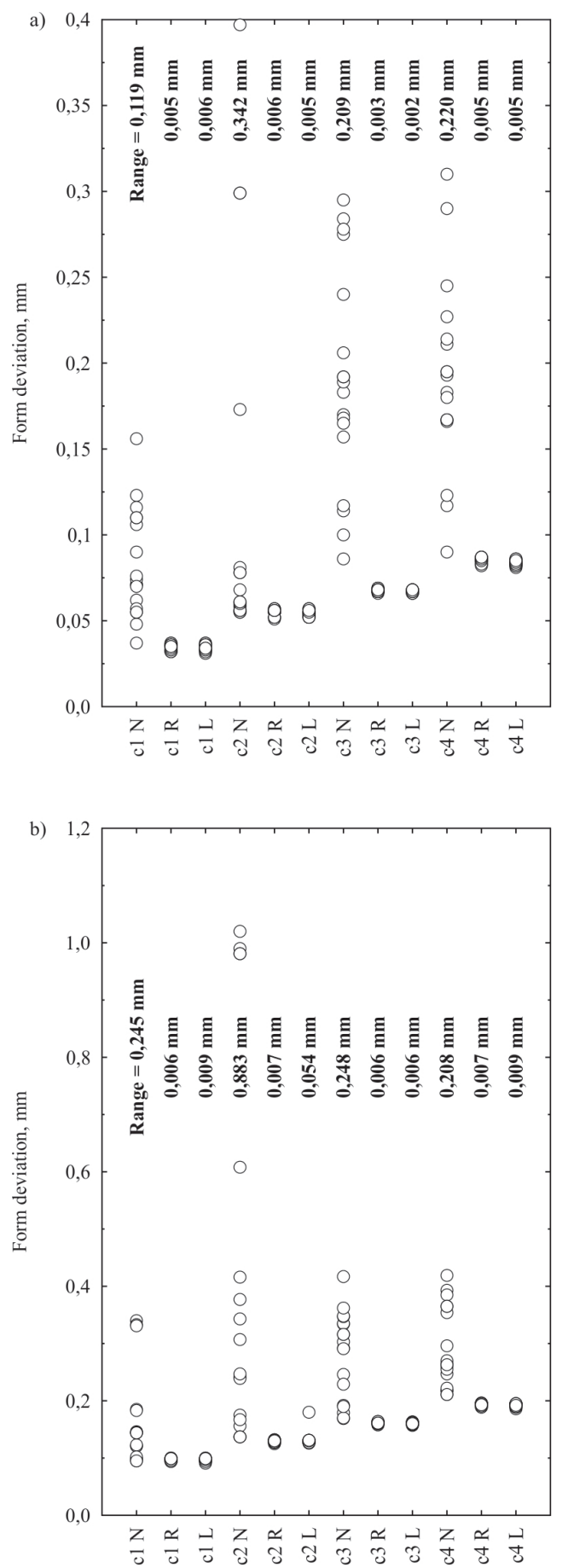

Figure 3 The influence of the method of form deviation calculation on the values of a form deviation in the case of: a) the first object, b) the second object

The algorithm is composed of several stages. The first stage consists in determining the approximated measured profile, which represents a cross-section of a workpiece. The measured curve is approximated by a polyline which passes through individual nominal points of a measured profile. Nominal points can be exported from the inspection software cooperating with a CMM when using the $2 \mathrm{D}$ curve measurement element. In the second stage lengths of parts of a polyline, denoted as $l_{i}$, are calculated. The lengths are determined as distances between subsequent nominal points. Then the following sums of the lengths are calculated: $l_{1}+l_{2}, l_{1}+l_{2}+l_{3}, \ldots, l_{1}+l_{2}+\ldots+l_{n-1}$, where $n$ is the number of nominal points. Those sums and the first length are stored in the respective set in order to be used for additional calculations. Moreover, the algorithm assumes that a stylus tip of a scanning probe moves along individual parts of a polyline using a uniform accelerated motion until the target speed is reached. Then a uniform motion is applied. The starting part of the polyline is the segment at which acceleration takes place. In the third stage, based on the assumed time of masking of measured points, the acceleration of the CMM and the scanning velocity, the distance travelled by a stylus tip of a probe is calculated. The next stage relates to the checking condition if the distance calculated in the previous step is greater than the individual elements of the set which were calculated in the second stage of the algorithm. In the case of fulfilment of that condition nominal points at which local deviations should not be taken into account when analysing the results of coordinate measurements are registered. Those nominal points can be eliminated by an operator of a CMM using the capabilities of the Calypso software.

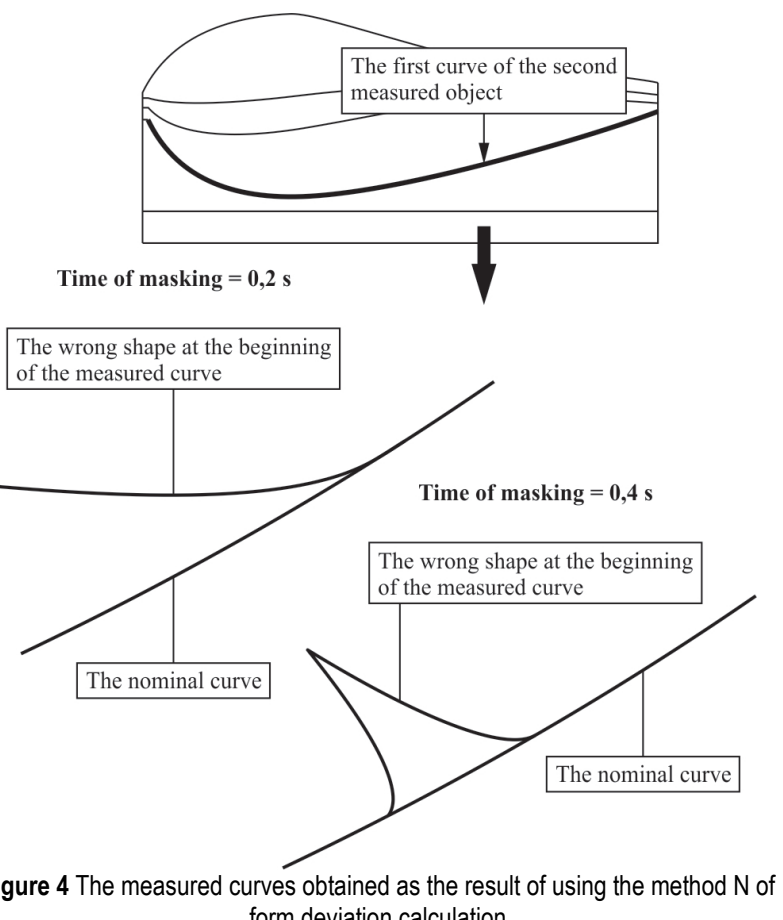

Figure 4 The measured curves obtained as the resu
form deviation calculation

In Tab. 1 the form deviations calculated with and without the use of the presented algorithm of the modification in combination with the method $\mathrm{N}$ are shown. Moreover, form deviations were calculated by using of the method R, which is based on measured points. The coordinate measurements were conducted for the first surface of the second workpiece. The measurements were performed in two different directions (Fig. 5). The radius of a stylus tip, analogously to the previous measurements, was equal to $1,5 \mathrm{~mm}$. The scanning velocity and the distance between measured points were equal to $15 \mathrm{~mm} / \mathrm{s}$ and $0,5 \mathrm{~mm}$, respectively. There were used the following values of time of masking of measured points: $(0,1 ; 0,2$; $0,3 ; 0,4 ; 0,5) \mathrm{s}$.

The smaller values of a form deviation calculated by using of the method $\mathrm{N}$ were achieved in the case of using the considered algorithm. The smaller deviations were gained due to the elimination of starting nominal points at which local deviations should not be calculated. That form deviations obtained using the proposed algorithm 
correspond to the deviations calculated using the method $\mathrm{R}$.

Table 1 The comparison of results calculated with and without the proposed algorithm in the case of the first curve of the second model

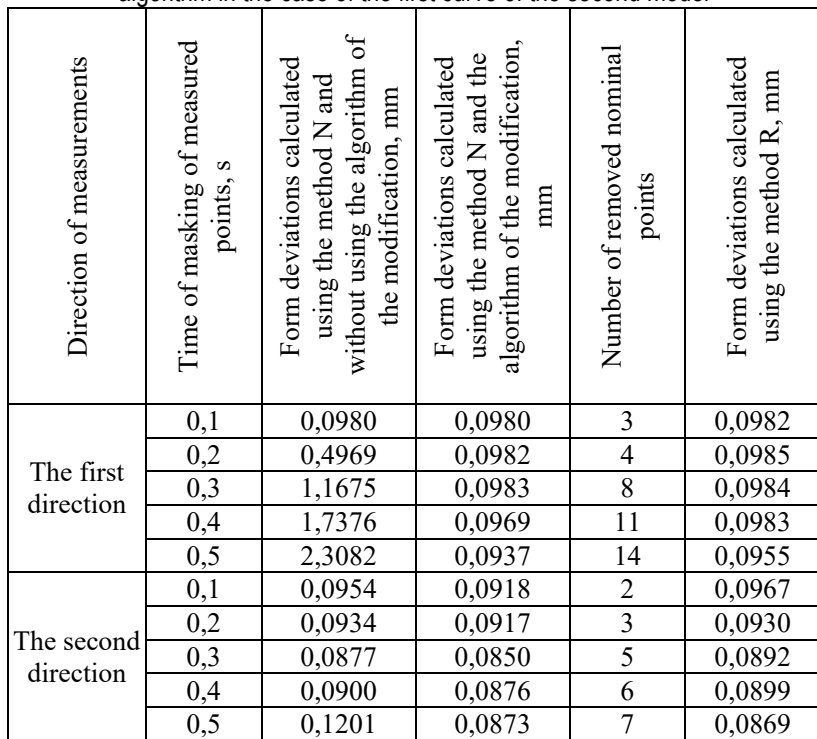

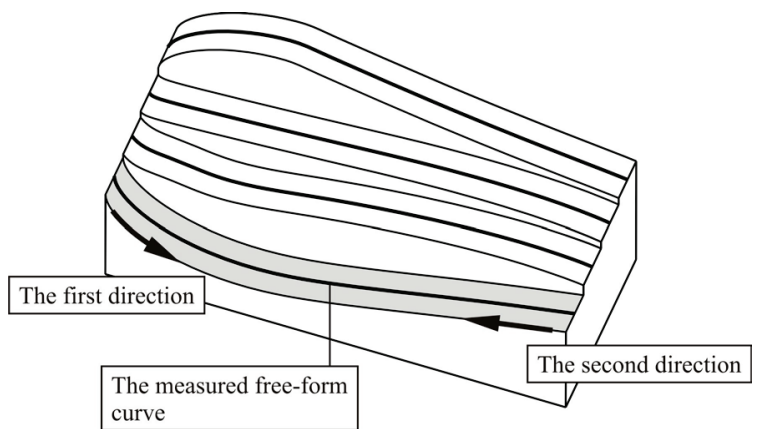

Figure 5 The directions of coordinate measurements of the first surface of the second object

\section{CONCLUSIONS}

Based on the results of experimental investigations it can be stated that the method using nominal points in the considered software is very sensitive for the used measurement parameters. This is the disadvantage of the method $\mathrm{N}$ which causes that it is very difficult to determine the quality of measured objects because the range of results obtained using that method is very large.

The proposed algorithm of the modification of a set of nominal points may support a user of a coordinate measuring system in the appropriate analysis of results of coordinate measurements of workpieces and increase the accuracy of form deviation calculation when using the method N. The developed algorithm may be included in the Calypso software by using of the PCM module. The results of measurements, which were obtained using the method $\mathrm{N}$ in combination with the algorithm of the modification of nominal points, correspond to the results gained using the method R.

The algorithm of deviation calculation based on the Lagrange interpolation method achieved better results of investigations than the method $\mathrm{N}$. The results obtained using the method $\mathrm{L}$ are close to the results gained by using of the reference method $\mathrm{R}$.

\section{REFERENCES}

[1] Kawalec, A. \& Magdziak, M. (2017). The selection of radius correction method in the case of coordinate measurements applicable for turbine blades. Precision Engineering, 49, 243-252. https://doi.org/10.1016/j.precisioneng.2017.02.012

[2] Yadong, L. \& Peihua, G. (2004). Free-form surface inspection techniques state of the art review. ComputerAided Design, 36(13), 1395-1417. https://doi.org/10.1016/j.cad.2004.02.009

[3] Poniatowska, M. (2012). Deviation model based method of planning accuracy inspection of free-form surfaces using CMMs. Measurement, 45(5), 927-937. https://doi.org/10.1016/j.measurement.2012.01.051

[4] ElKott, D. F. \& Veldhuis, S. C. (2005). Isoparametric line sampling for the inspection planning of sculptured surfaces. Computer-Aided Design, 37(2), 189-200. https://doi.org/10.1016/j.cad.2004.06.006

[5] ElKott, D. (2011). Coordinate metrology of freeform surfaces. A CAD-based, practical approach. Saarbrücken, VDM Verlag Dr. Müller GmbH \& Co. KG.

[6] ElKott, D. F., Elmaraghy, H. A., \& Elmaraghy, W. H. (2002). Automatic sampling for CMM inspection planning of free-form surfaces. International Journal of Production Research, 40(11), 2653-2676. https://doi.org/10.1080/00207540210133435

[7] ElKott, D. F. (2001). Automatic sampling for CMM inspection planning of free form surfaces. MSc thesis, Windsor, Ontario, Canada, The University of Windsor.

[8] Mingrang, Y., Yingjie, Z., Yunlong, L., \& Ding, Z. (2013). Adaptive sampling method for inspection planning on CMM for free-form surfaces. The International Journal of Advanced Manufacturing Technology, 67(9-12), 1967-1975. https://doi.org/10.1007/s00170-012-4623-0

[9] Barari, A. (2013). Inspection of the machined surfaces using manufacturing data. Journal of Manufacturing Systems, 32(1), 107-113. https://doi.org/10.1016/j.jmsy.2012.07.011

[10] Magdziak, M. (2016). An Algorithm of Form Deviation Calculation in Coordinate Measurements of Free-Form Surfaces of Products. Strojniški vestnik - Journal of Mechanical Engineering, 62(1), 51-59. https://doi.org/10.5545/sv-jme.2015.3039

[11] Calypso, software documentation. (2010). Oberkochen, Carl Zeiss Industrielle Messtechnik.

[12] Cookbook Measurement Strategies. (2015). Carl Zeiss Industrielle Messtechnik GmbH.

\section{Contact information}

Marek MAGDZIAK, PhD, Eng.

Rzeszow University of Technology,

The Faculty of Mechanical Engineering and Aeronautics,

Department of Manufacturing Techniques and Automation,

al. Powstańców Warszawy 12, 35-959 Rzeszów, Poland

E-mail: marekm@prz.edu.pl 